EPJ Web of Conferences 106, 05003 (2016)

DOI: $10.1051 /$ epjconf/201610605003

(C) Owned by the authors, published by EDP Sciences, 2016

\title{
Coded-Aperture Compton Camera for Gamma-Ray Imaging
}

Aaron M. Farber and John G. Williams ${ }^{\mathrm{a}}$

Aerospace \& Mechanical Engineering Department, University of Arizona, Tucson, AZ 85721, USA

\begin{abstract}
A novel gamma-ray imaging system is demonstrated, by means of Monte Carlo simulation. Previous designs have used either a coded aperture or Compton scattering system to image a gamma-ray source. By taking advantage of characteristics of each of these systems a new design can be implemented that does not require a pixelated stopping detector. Use of the system is illustrated for a simulated radiation survey in a decontamination and decommissioning operation.
\end{abstract}

\section{Introduction}

Gamma imaging has found many applications in nuclear physics, astronomy, medical science and national security, and has sometimes been employed in the nuclear power industry [1]. Current gammaray imaging systems utilize pixelated detectors in either Compton scattering or coded-aperture systems. In this paper, simulation of a new hybrid system is reported.

Coded-aperture masks were proposed in 1968 in papers by Dicke [2] and Ables [3]. They use multiple apertures in a manner analogous to pinhole imaging, together with image reconstruction techniques to decode the overlapping images from many apertures. A position-sensitive, or pixelated, photon detector is placed behind the coded-aperture mask.

Compton scattering imagers collect coincidence data from at least two events in a cascade from a Compton scattered photon [4]. In one version [5] the two detectors are a position-sensitive gamma scattering spectrometer that detects Compton electrons and a second full-energy spectrometer, also position sensitive, that detects the scattered photons. Since these are distributed in paths that form a conical projection onto the second detector, image reconstruction techniques are required [6].

The design demonstrated in this paper utilizes a thin pixelated silicon spectrometer, a coded-aperture mask, and a monolithic full-energy $\mathrm{NaI}(\mathrm{Tl})$ scintillation detector behind the coded aperture [7]. This configuration provides the same angular resolution and wide field-of-view as a conventional Compton camera with the same geometry, without requiring a second pixelated detector. This also results in considerable simplification of the data acquisition and data processing systems.

The principal advantage of the configuration presented here is that thick, monolithic, full-energy spectrometers (e.g. NaI, CsI, $\mathrm{LaBr}$ ) can be used to provide detection efficiencies, up to $1500 \mathrm{keV}$, that exceed $90 \%$, with peak-to-total ratios of $>70 \%$ and are available in standard configurations with cylindrical dimensions up to 10 inches in diameter and length [8]. No pixelated spectrometer with such efficiencies is commercially available.

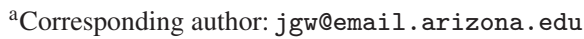

This is an Open Access article distributed under the terms of the Creative Commons Attribution License 2.0, which permits unrestricted use, distribution, and reproduction in any medium, provided the original work is properly cited. 
(a)

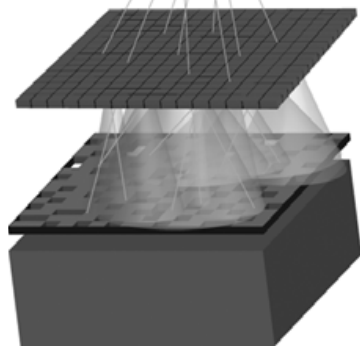

(b)

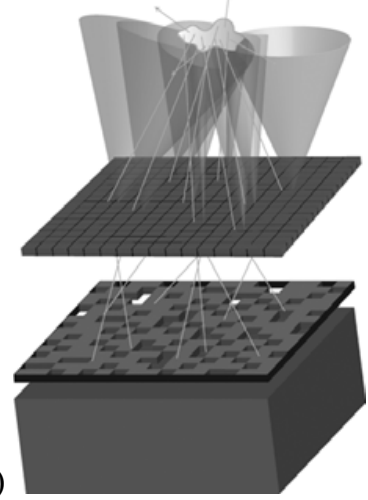

Figure 1. Schematic of proposed detector showing (a) forward, and (b) adjoint transport of photons through the system.

Other designs have been proposed that combine the use of Compton scattering with a mask, but in a different fashion. Some simply use a collimator type mask, which serves to isolate the direction of the incoming photon [9]. Although this significantly helps in the reconstruction of the source location, it comes at great cost to efficiency. Other systems utilize a coded-aperture mask placed in front of the first detector [10]. This allows the camera to operate as either a coded aperture or Compton camera, as well as in a hybrid mode where the coded aperture serves to limit the potential directions a particular photon could take. However, this system still employs two pixelated detectors.

\section{Geometry \& Materials}

The geometry of the system modelled here consists of a Compton detector layer, $100 \mathrm{~mm}$ square in area and $2 \mathrm{~mm}$ thick, positioned $50 \mathrm{~mm}$ above a coded-aperture mask that covers the full-energy detector. The design concept for the system is illustrated in Fig. 1. Incident photons scatter in the Compton layer, and those of a specific scattered energy are distributed in cones with a specific scattering angle, with random dihedral angle distributed uniformly on 0 to $2 \pi$. By measuring the scattered photon's energy, its scattering angle can be determined. This concept of the forward problem can be seen in Fig. 1(a) as photons propagate through the system.

In a conventional two-photon Compton camera, the position of the photons that arrive at the second layer must be determined. It is also important that the second detector layer has a high probability of recording full-energy events. The source directions and energies can then be reconstructed. In our system, however, the pixelated or position-sensitive full-energy detector is replaced by a single monolithic one, supplemented by a coded aperture placed over it. In previous coded aperture systems $[2,3]$, the mask is placed in front of a pixelated detector and multiple, overlapping, pinhole images are created. With the pattern of the aperture known, one can deconvolve the image data and reconstruct an image with much higher efficiency than with a single pinhole. In our design, the positions of the pixelated detector, in the Compton layer, and the coded aperture are reversed and we have added a subsequent, non-pixelated, energy absorbing detector. The same reconstruction technique would still work to produce an image of the scattered photons, and hence of their possible paths prior to scattering. This is illustrated in Fig. 1(b), which shows some of the back-projected, or conjugate paths.

For a successful spectrometry and imaging system, the full-energy detector must have a high probability of recording the full photon energy (subject to its resolution). In our system, the full-energy detector is not pixelated, but each recorded photon is in coincidence with a known Compton layer pixel. 


\section{$15^{\text {th }}$ ISRD}

Therefore, the information about its possible direction is equivalent to that obtained in a conventional coded aperture camera with the same coding pattern. We know that if an adequate sample of photon energies and pixel coordinates is obtained, with a suitable mask pattern, the energy- and angle-dependent photon flux leaving the Compton layer can be found, particular to each pair of energies recorded in the two detectors. Thus the coded aperture system provides the needed estimate of the scattered photon flux in each direction that falls within the square aperture of the mask.

We chose to consider coded aperture masks that are based on a square lattice of the same dimensions as the pixelated Compton detector. This choice ensures that the ray pencils formed between the pixels of the latter and the cells of the former are analogous to the principal lattice directions of a rectangular crystal. Thus the same scattered photon directions are repeatedly sampled by different paths through the system. We also chose to consider mask patterns with $50 \%$ of the cells open. This choice is the one that maximizes the count rate, which is the best choice for detection of features at low count rates, though other choices may be optimal in some circumstances [11]. The penalty for using many open channels in the mask is the increased ambiguity about the path taken by each single photon. Reconstruction of the angular distribution can amplify the statistical noise. The selection of a suitable mask pattern is needed to minimize this effect [12].

Thus, two types of reconstruction are implied: one comparable to that used in a Compton camera with two layers of pixelated photon detectors, and the other comparable to that used in a coded-aperture camera with a single pixelated detector and a coded aperture. In our actual reconstruction scheme the reconstruction is done as a single step.

\section{Simulation Method}

In the simulation, Compton scattering occurs at a single plane, where only single scattering events are considered. At energies above $1.022 \mathrm{MeV}$, pair production in the Compton layer should be considered. By referring to NIST XCOM data [13] it can be seen that even up to $1.5 \mathrm{MeV}$, pair production contributes only about one-third of one percent of the events. For this reason we did not include it in our simulations.

The planned size of the Compton detector is $100 \mathrm{~mm}$ square, on a $150 \mathrm{~mm}$ diameter wafer, with a thickness of $2 \mathrm{~mm}$. Analysis has shown that for a silicon detector $2 \mathrm{~mm}$ thick, the interaction efficiency, the fraction of incident photons that scatter, ranges from $2.39 \%$ at $1.5 \mathrm{MeV}$ to $6.53 \%$ at $150 \mathrm{keV}$ for normally incident photons and $4.72 \%$ at $1.5 \mathrm{MeV}$ to $12.6 \%$ at $150 \mathrm{keV}$ for photons incident at an angle of radians from the normal. At these interaction rates, only considering single scattering events is a reasonable approximation.

The coded-aperture mask material is a high-density material, such as a tungsten alloy. The simulation assumes an ideal mask, where all photons that hit the closed portions are completely blocked. In a fully realized model, the mask must be thick enough to stop a substantial fraction of the photons incident on the closed portions and thin enough to avoid acting as a collimator. This may dictate the distance required between the detectors as well as their size. An advantage of this geometry, with the mask behind the Compton detector, is that scattered photons incident on the mask are already lower in energy than the primary photons.

The imaging theory uses the equation below (1),

$$
\langle I\rangle=H . O
$$

where $I$ is the image data in vector form, $O$ is the object in vector form, and $H$ is a matrix containing the conditional probabilities:

$$
H=\left[p\left(I_{i} \mid O_{j}\right)\right]
$$


The image vector, $I$, is made up of four attributes, two for energy, each with 16 bins, and two for position, each with 64 bins. The four attributes are energy in the Compton layer, energy in the full-energy detector layer and position in each layer. Despite the design described here not having position information in the full-energy detector, the simulation retains this information for validation and debugging as well as to compare results and performance to a theoretical traditional two-photon Compton camera. Thus, the image vector contains $2^{20}$, or approximately $1 \mathrm{M}$ entries.

We have implemented several versions with differing spatial resolution. For the results presented here, the object vector, $O$, contains 256 spatial bins with 16 energy bins for each. The spatial bins are determined by equally spaced angles from $-\pi / 3$ to $\pi / 3$ in both the $\mathrm{x}$ and $\mathrm{y}$ direction. The energy bins are equally spaced from $150 \mathrm{keV}$ to $1.45 \mathrm{MeV}$.

The program simulates each photon through ray tracing. To generate the imaging matrix, $H$, each possible incoming direction and energy is simulated several million times. The program cycles through each spatial pixel of the Compton detector, selecting random interaction points within the pixel. It then samples the probability distribution to select an outgoing direction and corresponding energy. Its projection on to the coded-aperture mask plane is then determined and the event is scored in the appropriate cell of the coded-aperture plane (whether occluded or not). This maps into a column of the $H$ matrix as probabilities of a given object photon creating a specific image event. Each column of the $H$ matrix is a vector of the probabilities of a given image conditional on a specific location and energy in object space, while each row is a vector proportional to the likelihood of a given object event, given a specific image event. With the number of pixels and energy bins growing, the dimensions of the $H$ matrix quickly grow to sizes difficult to manipulate even with powerful computers. Although this matrix only needs to be generated once, it requires the bulk of the computation time needed to simulate the system. More details on the $H$ matrix can be found in Foundations of Image Science [14].

In the simulations reported here, $H$ is a $(1 \mathrm{M} \times 4 \mathrm{k})$ matrix. When the coded aperture is used, all of the counts in the open half ( 32 out of 64 ) of the full-energy position bins are summed into a single bin. This reduces the dimension of the $H$ matrix to $(16 \mathrm{k} \times 4 \mathrm{k})$.

The test object is run through the same Monte Carlo, but with its specific locations, source strengths and energies. These results are then run through a reconstruction computation. Reconstructions were done with both least squares estimation (LSEM) and maximum likelihood (MLEM), but the former were unsatisfactory because the large dimension of the object vector (16k) ensures that the number of counts in each bin is too small to exhibit Gaussian statistics.

All Monte Carlo simulations were built within Matlab. The modular design of the code allows for quick changes in all design parameters, such as numbers of pixels, energy bins and relative distances between detectors. Other Monte Carlo programs, such as Geant [15] or MCNP [16] could be used to implement the simulation, though the scoring scheme is somewhat cumbersome to model. Building a code for this specific purpose simplifies the rapid testing of multiple configurations without significant time spent reprogramming the geometry. The Compton scattering cross sections in the simulation are governed by the Klein-Nishina formula [17]. In order to incorporate the appropriate scattering crosssections into the model, we found the solution used in EGS4 [18] to be very efficient and easy to implement.

The coded aperture simulated was modelled as ideal. This means it has no thickness and is $100 \%$ opaque to all photons that strike the closed portions.

Binned data acquisition was used with $64 \times 64$ pixels, for the two detection layers, together with 4-bit energy resolution, in each layer. Much larger arrays can be used with list-mode data acquisition/image reconstruction $[19,20]$. Various $50 \%$ open mask patterns were tested to find an effective pattern. Other masks, such as Uniformly Redundant Arrays (URAs) [21] and Modified URAs (MURA) [22] should also be considered. Figure 2 shows the mask in the work presented here. 


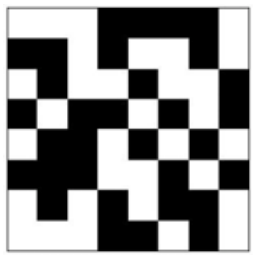

Figure 2. Example of an $8 \times 8$ mask pattern, used in simulations presented here.

\section{Reconstruction Results}

The principle advantage of simulating and scoring the location information from the full-energy detector is that a traditional two-photon Compton camera can be simulated to compare results. The imaging performance of the traditional Compton camera and the coded-aperture version were tested in their $4 \times 4$ pixel configuration using the input test object shown in Fig. 3(a).

Because of the object's 2-dimensional position and 1-dimensional energy, the choice was made to map the object space in a way that could allow for this 3-dimensional data to be displayed in a single, 2-dimensional image. For this reason, it was decided to represent the object space with a mapping, where each position pixel is subdivided into 16 pixels representing the various energy bins. Since the "A" object is unrealistic, this imaging example serves to demonstrate the encoding and decoding of the information from object to reconstructed image. For real-world imaging examples, energy spectra are displayed within each spatial pixel to provide a more intuitive picture.

In this paper the number of events, $\mathrm{N}_{\mathrm{i}}$, in the test objects is the number of photons detected in the Compton layer, while the number of events, $\mathrm{N}_{\mathrm{o}}$, in the reconstructions are the number of events also recorded in the full-energy detector and recorded in the image space. These are not the same, because not all photons scattered in the Compton layer reach the full energy detector. The source strength of a given number of photons detected can be calculated using the absolute efficiency of the detector.

Initially the simple least squares (LS) estimator (3) was used:

$$
\hat{O}=\left(H^{\prime} \cdot H\right)^{-1} H^{\prime} \cdot I .
$$

Upon further testing, the MLEM estimator [23] proved to yield similar results at high count rates, and more accurate results at lower count rates. MLEM requires the implementation of an iterative algorithm:

$$
\hat{f}_{j}^{k+1}=\frac{\hat{f}_{j}^{k}}{S_{j}} H_{j}^{\prime}\left(\frac{I}{H \cdot \hat{f}^{k}}\right), \quad \text { for all } j
$$

where $\hat{f}_{j}^{k}$ is the $k^{\text {th }}$ iteration of the reconstructed object vector, $I$ is the collected image data, $S_{j}$ is a normalizing term, and the subscript $j$ indicates the $j^{\text {th }}$ object voxel. In the term in brackets, the division of $I$ is an element-wise division while the multiplication in the denominator is a standard matrix multiplication.

In Figs. 3(b) and 3(c), the figures compare the performance of a conventional two-photon Compton camera with that of our coded-aperture system. Figures 3(b) is the image created using the MLEM reconstruction of a hypothetical pixelated full-energy detector, instead of the coded-aperture mask. Figures 3(c) shows the MLEM reconstructions from the coded-aperture version, applied to the same image data. The masked version can reconstruct a comparable image only when more counts are recorded. It should be remembered, however, that the efficiency actually achievable with a pixelated full-energy detector is much lower than that of a thick non-pixelated second detector. The comparison in Figs. 3(b) and 3(c), however, does not take this into account. 


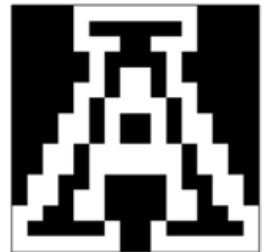

(a)

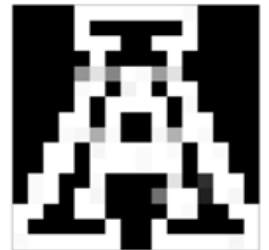

(b)

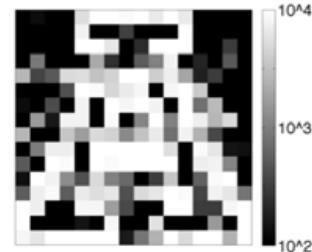

(c)

Figure 3. (a) Input test object $\left(\mathrm{N}_{\mathrm{i}}=1.06 \times 10^{6}\right)$ at medium count rate and MLEM reconstruction of (b) Compton camera $\left(\mathrm{N}_{0}=235099\right)$ and (c) coded Compton camera $\left(\mathrm{N}_{0}=117891\right)$.

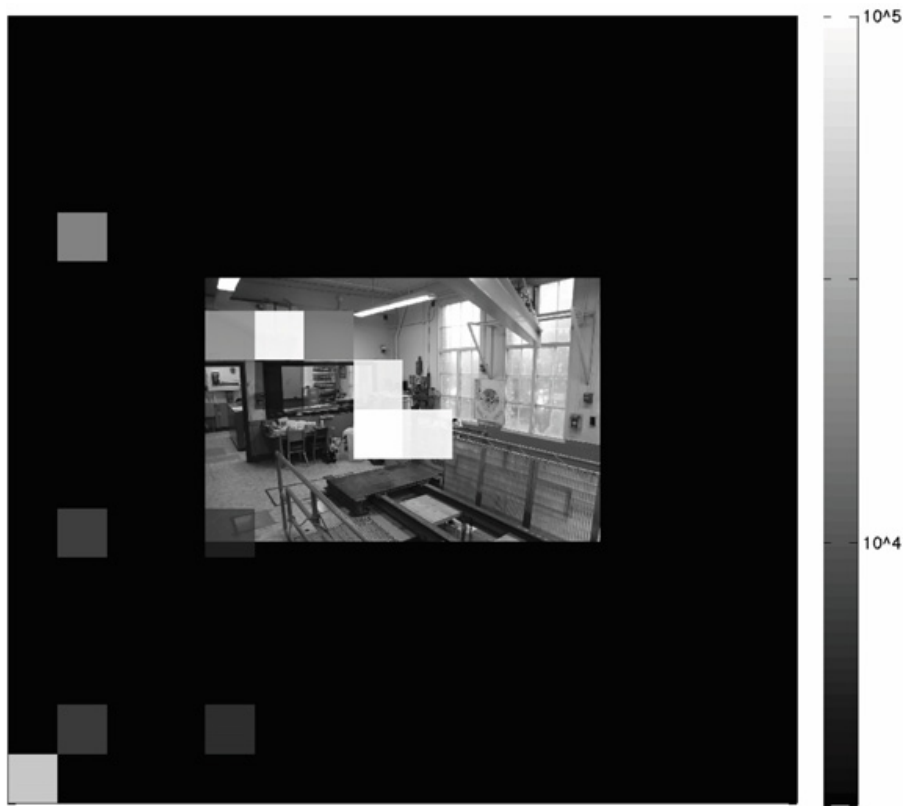

Figure 4. The field of view of the gamma imaging system is represented by the black square, which corresponds to an angular aperture of 120 degrees in height and width. A photographic image is superimposed at the center. This corresponds to the field of view of a $28 \mathrm{~mm}$ focal length lens in $35 \mathrm{~mm}$ format. Superimposed on the whole are the total intensities of the reconstructed photon energy fluence. The gray scale (shown at the right) has units of $\mathrm{MeV}$ per pixel incident on the detector area. The intensity is only shown for those pixels in which the threshold fluence of $3 \times 10^{3} \mathrm{MeV}$ is exceeded. The two simulated point sources have activities of $3.6 \mathrm{mCi}\left(1.33 \times 10^{8} \mathrm{~Bq}\right){ }^{137} \mathrm{Cs}$ (upper left of the photo image) and $1.2 \mathrm{mCi}\left(4.53 \times 10^{7} \mathrm{~Bq}\right){ }^{60} \mathrm{Co}$ (center of the photo image).

\section{Detection of Point Sources}

The current Monte Carlo simulation does not directly calculate the systems' detection efficiency because the full-energy detector is not modelled, but is treated as a black detector of full-energy events. Also, the first interaction probability in the Compton layer is not calculated, since only incident photons that undergo a Compton scatter are considered. These factors are relatively easy to estimate, however, using simple assumptions.

When considering the efficiency for detection of ${ }^{137} \mathrm{Cs}$ gamma rays, at $662 \mathrm{keV}$, the following approximate values can be used. Full-energy detection of Compton scattered photons, for energies less than $662 \mathrm{keV}$ can easily have a full-energy efficiency of 70\%, with the use of a large scintillator crystal. The Compton efficiency of a $2 \mathrm{~mm}$ thick silicon detector, for normally incident photons at $662 \mathrm{keV}$, is 


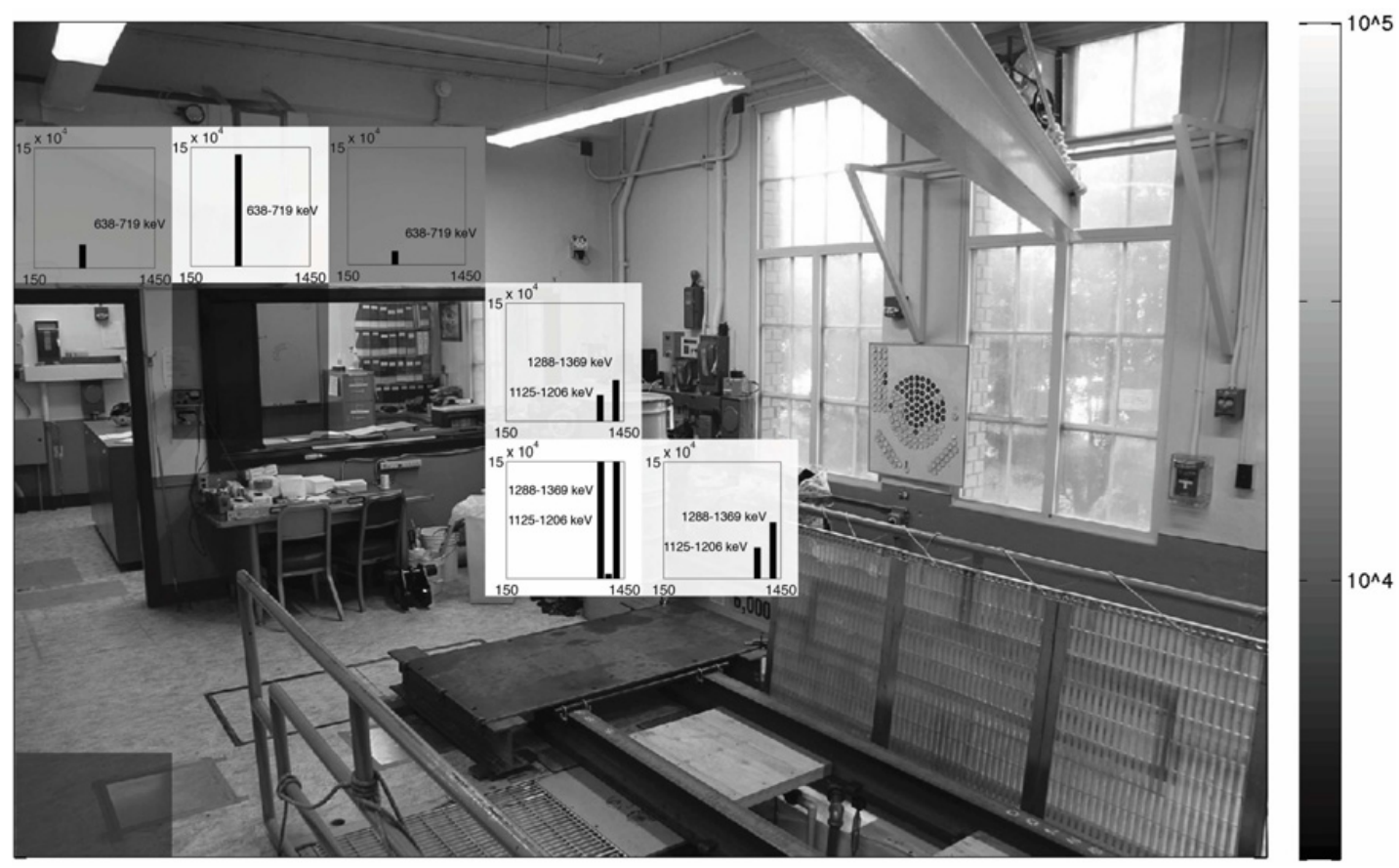

Figure 5. Zoomed in view of the same result as Fig. 4, with spectra (binned in 16 energy intervals) of the detected source reconstructions. The point sources were actually located within the two pixels in which the greatest intensity was reconstructed. Each pixel represents an angular area of $7.5^{\circ} \times 7.5^{\circ}$.

$3.4 \%$. Our simulation gives an efficiency of $12.2 \%$ for detection of a Compton-scattered photon in the full-energy detector through the mask pattern. Combining these values, we find an intrinsic efficiency of the system, for normally incident ${ }^{137} \mathrm{Cs}$ photons, of $2.9 \times 10^{-3}$.

The geometrical efficiency of a $100 \mathrm{~cm}^{2}$ detector, for a point source at $10 \mathrm{~m}$ is $(1 / 4 \pi) \times 10^{-4} \cong$ $8.0 \times 10^{-6}$. Thus, the overall efficiency for detection of a point source of ${ }^{137} \mathrm{Cs}$ at a distance of $10 \mathrm{~m}$ is $2.31 \times 10^{-8}$. The count rate from a $10 \mathrm{mCi}\left(3.7 \times 10^{8} \mathrm{~Bq}\right)$ source would be 8.5 counts per second, or 5130 counts in 10 minutes. Identification of a point source, with high confidence, could be achieved with very few counts, however, depending on background. The preceding calculation does not account for attenuation in air. At a distance of $10 \mathrm{~m}$, this corresponds to a transmission factor of approximately $90 \%$. The local dose rate, from directly transmitted gamma rays, at a distance of $10 \mathrm{~m}$ from a $10 \mathrm{mCi}$ $\left(3.7 \times 10^{8} \mathrm{~Bq}\right)$ source of ${ }^{137} \mathrm{Cs}$ is approximately $0.3 \mu \mathrm{Sv} / \mathrm{hr}(0.03 \mathrm{mrem} / \mathrm{hr})$. This can be compared with an average gamma background rate of $0.1 \mu \mathrm{Sv} / \mathrm{hr}$, which is approximately $80 \mathrm{mrem} / \mathrm{yr}$, a typical natural photon exposure level [24]. For the $7.5^{\circ}$ by $7.5^{\circ}$ square pixel in this system, the signal to noise ratio enhancement is approximately $730: 1$, for point sources relative to an omni-directional background.

Reconstruction of two point sources can be seen in Fig. 4 and Fig. 5. The reconstructed photon fluence is shown as an overlay on a photographic image, which has much smaller field of view. The image is of a research reactor room during decommissioning. Objects in the photo include a radioactive material shipping container and an exhaust duct that has a HEPA filter at its entrance. These locations coincide with the positions of the two simulated point sources, though such sources were not actually present at those locations. $\mathrm{A}{ }^{137} \mathrm{Cs}$ point source of activity $3.6 \mathrm{mCi}\left(1.33 \times 10^{8} \mathrm{~Bq}\right)$ was simulated at a distance of 7.5 meters, corresponding with the HEPA filter (upper left of image). For this source strength $10^{4}$ photons would be detected in the Compton layer. Also simulated was a $1.2 \mathrm{mCi}\left(4.53 \times 10^{7} \mathrm{~Bq}\right){ }^{60} \mathrm{Co}$ 
source at a distance of 6.0 meters, corresponding with the shipping container (image center), with $10^{4}$ photons detected in the Compton layer split between the two energies 1173 and $1332 \mathrm{keV}$. The number of coincidence events detected was about 1000 for each source. These numbers of events correspond to a counting time of 10 minutes. In this image, the gray-scale range was spaced logarithmically in total energy fluence intensity.

\section{Conclusions}

The simulation presented here includes some idealizations that cannot be actually realized. The most significant of these is the assumption of an ideal mask and of a black, full energy detector. Inclusion of these effects will tend to degrade the system performance at the highest part of the energy range. Energy resolution of the detectors is not modelled, but the energy bin width used is large compared to attainable energy resolutions. Thus this approximation has little effect. Chance coincidences could limit the environmental radiation level in which a device could be used. Heavy shielding of the full-energy detector is necessary. All of the effects mentioned will tend to produce a fogging of the image, compared to the examples shown.

The simulation of point sources has demonstrated the potential of the system to correctly identify isotropic sources, and locate their correct position within an angular area of $7.5^{\circ} \times 7.5^{\circ}$. Sources with activities of a few $\mathrm{mCi}$ are easily imaged from distances up to $10 \mathrm{~m}$. The source strengths used here appear to exceed by far the detection limit, but that limit cannot be properly determined until an actualization of the system is built and tested. The system appears to have the ability to perform useful radiological scanning during decontamination and decommissioning and other types of survey.

The authors would like to thank Dr. Barry Ganapol and the funding from his Nuclear Energy University Programs (NEUP) grant for providing us with access to the GPU computing platform. Additionally, we give big thanks to Dr. Lars R. Furenlid for his support and guidance.

\section{References}

[1] N. E. Royle, and R. D. Speller, IEEE Nuclear Science Symposium, 1, 365-368 (1997)

[2] R. H. Dicke, Astrophysical Journal, 153, L101 (1968)

[3] J. G. Ables, Proceedings of the Astronomical Society of Australia, 1, 172 (1968)

[4] L. E. Peterson, and R. L. Howard, Nuclear Science, IRE Transactions on, 8, 21-29 (1961)

[5] V. Schönfelder, A. Hirner, and K. Schneider, Nuclear Instruments and Methods, 107, 385-394 (1973)

[6] A. J. Rockmore, and A. Macovski, IEEE Trans. on Nuclear Science, 23, 1428-1432 (1976)

[7] A. M. Farber, and J. G. Williams, IEEE Nuclear Science Symposium and Medical Imaging Conference (NSS/MIC), 450-454 (2012)

[8] Saint-Gobain Crystals, Efficiency Calculations for Selected Scintillators [Brochure] (2004-8)

[9] Z. He, et al. Nuclear Science, IEEE Transactions on, 42, 668-674 (1995)

[10] L. E. Smith, et al. IEEE Nuclear Science Symposium, 2, 1040-1045 (1998)

[11] J. J. M. in 't Zand, J. Heise, and R. Jager, Astronomy and Astrophysics, 288, 665-674 (1994)

[12] G. K. Skinner, Nuclear Instruments and Methods in Physics Research: Proceedings of the International Workshop on X- and $\gamma$-Ray Imaging Techniques, 221, 33-40 (1984)

[13] M. J. Berger, et al. XCOM: Photon Cross Section Database (version 1.5), (2010)

[14] H. H. Barrett, and K. Myers, Foundations of Image Science (2004)

[15] J. Allison, et al., Nuclear Science, IEEE Transactions on, 53, 270-278 (2006)

[16] X-5 Monte Carlo Team, MCNP - A General N-Particle Transport Code, Version 5 - Vol 1: Overview and Theory (2005) 


$$
15^{\text {th }} \text { ISRD }
$$

[17] O. Klein, and T. Nishina, Zeitschrift für Physik A Hadrons and Nuclei, 52, 853-868 (1929)

[18] W. R. Nelson, H. Hirayama, and D. W. O. Rogers, THE EGS4 CODE SYSTEM, SLAC-0265, (1985)

[19] H. H. Barrett, T. White, and L. C. Parra, J. Opt. Soc. Am. A, 14, 2914-2923 (1997)

[20] L. Parra, and H. H. Barrett, IEEE Transactions on Medical Imaging, 17, 228-235 (1998)

[21] E. E. Fenimore, and T. M. Cannon, Appl. Opt., 17, 337-347 (1978)

[22] S. R. Gottesman, and E. E. Fenimore, Appl. Opt., 28, 4344-4352 (1989)

[23] C. L. Byrne, Image Processing, IEEE Transactions on, 2, 96-103 (1993)

[24] United Nations Scientific Committee on the Effects of Atomic Radiation, Sources and effects of ionizing radiation: United Nations Scientific Committee on the Effects of Atomic Radiation: UNSCEAR 2010 report to the General Assembly, with scientific annexes. 2008 